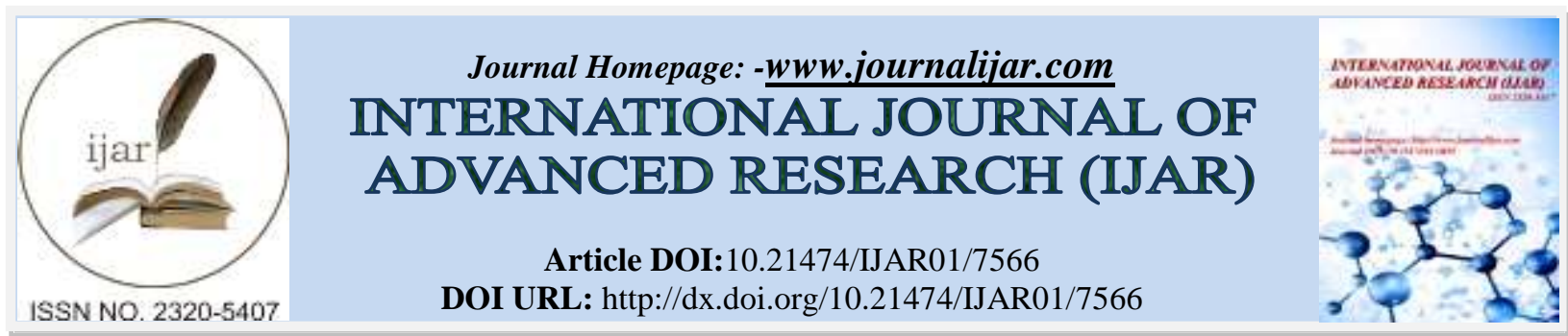

RESEARCH ARTICLE

\title{
EFFECT OF SWIMMING AS A HYDROTHERAPEUTIC INTERVENTION FOR THE MANAGEMENT OF CHRONIC NON-SPECIFIC LOW BACK PAIN - AN EXPERIMENTAL STUDY.
}

\author{
Subhasmita Dwivedy ${ }^{1}$, Dr. PatitapabanMohanty, Ph. D., Assoc. Prof. (PT) ${ }^{2}$ and Mrs. MonalisaPattnaikand \\ MPT, Asst. Prof. (PT) ${ }^{2}$. \\ 1. Swami Vivekanand National Institute of Rehabilitation Training. \\ 2. Research, Olatpur, Bairoi, Cuttack, India.
}

\section{Manuscript Info}

Manuscript History

Received: 15 June 2018

Final Accepted: 17 July 2018

Published: August 2018

Keywords:-

Hydrotherapy, swimming, chronic nonspecific low back pain, aquatic therapy.

\begin{abstract}
Objective: to evaluate the effect of swimming, as a hydrotherapeutic intervention, in subjects with chronic non specific low back pain, in reducing pain, improving range of motion and function within a period of four weeks and analyzing the post therapeutic effect on the same outcome measures following four more weeks of non intervention by performing extensive experimental investigation. Design: experimental study Setting: Hydrotherapy unit of Department of Physiotherapy,SVNIRTAR Participants: 51 subjects suffering from chronic non-specific low back pain Interventions: The exercises employed consisted of walking in water and aquatic aerobics as a warm up regimen of 10 minutes followed by swimming for thirty minutes and then Ai Chi technique of relaxation as a cool down regimen for another 10 minutes. All the subjects participated in the exercise program for 4 weeks. The frequency of performing exercises was five days a week. Outcome Measures: VAS, Modified Schober's Test, Quebec Back Pain Disability Scale. Results: Statistically significant improvements were detected in all outcome measures compared with baseline, after 4 weeks of intervention. The improvement in physical score was independent of the initial ability in swimming. The post scores taken after 4 weeks of non-intervention noticed a mild change in the statistical values of VAS and Quebec Questionnaire. Conclusions: The results obtained suggested that swimming can be recommended for the treatment of chronic non specific low-back pain as it is useful in not only reducing pain but also improving the range of motion and the function of the individual.
\end{abstract}

Copy Right, IJAR, 2018,. All rights reserved.

\section{Introduction:-}

In today's advanced technology era, people prefer to use technology induced luxuries instead of doing physical work. Relatively reduced physical activity results in many discomforts, low back pain being one of the many.

Hoy D, March L et al.on March, 2012 led the latest Global Burden Of Disease Study and found low back pain and osteoarthritis are now ranked $2^{\text {nd }}$ only to cancer as a leading cause of disease burden in Australasia and low back 
pain being the highest contributor to disability in the world, according to a pivotal international study released on December 14, 2012.[1]Low back pain is a multi factorial disorder with many possible etiologies. It has been reported that $60-80 \%$ of people in the Western hemisphere suffer from back pain at some time in their life. [2] Of these, $85 \%$ are classified as suffering from non-specific low back pain (NSLBP). [3]

Chronic Non-specific low back pain is defined as persistent and disabling low back pain not attributable to a recognisable, known specific pathology lasting for more than three months. [4, 5]

In the acute phase, the pain is likely to be elicited by structural changes which are not necessarily identifiable, and may be associated with structural impairments in the back. However, unlike most health problems, such specific pathophysiology is often not found in chronic low back pain. Pain symptoms and the associated disability are often associated only weakly with clinical findings and measures of loss of functioning. [6] Prospective studies have demonstrated that the experience of back pain is characterized by change and variation through recurrences and remissions across pain episodes, making accurate diagnosis all the tougher. [7]

Endurance of lumbar stabilizer is most important key for preventing lumbar pain.Trunk muscle endurance training has been recommended as means of increasing fatigue threshold and improving performance and reducing disability. That is why exercise therapy is the base of treatment and rehabilitation of CNSLBP. [8]

With the increasing prevalence of low back pain in the Indian population, which has been found to range from $6.2 \%$ to $92 \%$. [9] More no. of studies regarding the treatment of the same is the need of the time.

Since the earliest recorded history, water has always been believed to promote healing and has therefore been widely used in the management of medical ailments. Aquatic immersion has profound biological effects, extending across essentially all homeostatic systems.Aquatic therapies are beneficial in the management of patients with musculoskeletal problems, neurologic problems, cardiopulmonary pathology, and other conditions. In addition, the margin of therapeutic safety is wider than that of almost any other treatment milieu. [10]

Nearly all the biological effects of immersion are related to the fundamental principles of hydrodynamics. The essential physical properties of water that effect physiologic change are density and specific gravity, hydrostatic pressure, buoyancy, viscosity, and thermodynamics.

An aquatic exercise program may be designed to vary the amount of gravity loading by using buoyancy as a counterforce. They, however, offer the ability to damp the force of movement instantaneously because of the viscous properties of water.Offloading of body weight occurs as a function of immersion, but the water depth chosen may be adjusted for the amount of loading desired. [11] The spine is especially well protected during aquatic exercise programs, which facilitates early rehabilitation from back injuries.

Hydrotherapeutic intervention, especially a high intensity, low impact exercise like swimming has been seen to decrease spasm, induce relaxation, improve range of motion, decrease physical disability, strengthen core muscles, strengthen extensor group of muscles, reduce pain in arthritis and fibromyalgia, cardio respiratory conditioning, treat pain and psychiatric rehabilitation, obesity management etc. [12-18] All of which have significant roles in chronic nonspecific low back pain.

Methodology:-

Type of study:-experimental

Research design:-A pre-test, post-test structured study design

\section{Inclusion Criteria:-}

Total sample consisted of 51 subjects (10 females and 41 males) within the age of 18 - 30 years, (mean age 23. 3) with chronic non specific low back pain characterized by a chief complaint of low back pain since the last three months or more. Pain not attributable to a recognized known specific pathology e.g. infection, tumor, osteoporosis, fracture, structural deformity, inflammatory disorder, radicular syndrome or cauda equine syndrome etc. Pain might be associated with mechanical factors like occupational sitting, backward postures, standing /walking bending twisting lifting, carrying but not independently. The outcome of acute spells is obscured by frequent relapses. Both genders were included in the study, may or may not know swimming. 


\section{Exclusion criteria:-}

Any cognitive deficit, uncooperative patient, acute organic neurologic deficit, neoplastic or inflammatory lesion, acute febrile infections, skin suppuration, Chlorine or bromine allergy, Serious fear of water, COPD or other similar respiratory issues, previous back surgery.

\section{Procedure:-}

The group of 51 subjects comprising of 10 females and 41 males with continuing CNSLBP for an average of 8.6 months, were explained about the study. Informed consent was taken prior to any treatment of interested subjects. The base scales of VAS, modified schober's test, Quebec Back Pain Disability Scale were taken. The intervention comprised of three steps as follows:

First a warm up regimen which included walking in water, then a few ROM exercises of the upper limb and lower limb combined with aerobic exercises like hacky sack, hop scotch, jumping jack, cross country ski, frog lunge jump, squat jump inside water and those using swimming boards. Each exercise was performed for ten repetitions and the whole warm up regimen was done for 10 minutes.

The second step involved swimming in freestyle mode along the length of the pool for the next half an hour ,taking adequate rest in between consecutive rounds. Those who did not know swimming were asked to follow the movement patterns of the upper limbs using pool noodles followed by the movement patterns of the lower limb holding onto the side of the pool, then practicing those movements together using two noodles in shallow side of the pool, till they learnt swimming without the help of noodles/ floats.

Third step included a cool down regimen where subjects were asked to do a slow jumping inside water followed by Ai Chi exercises regimen for 10 minutes.

Then at the end of four weeks, recording of changes in post treatment pain on VAS, ROM measure by modified scober's test and QBPDS score were taken.

Then a period of non intervention for another four weeks time was given. At the end of these four weeks, recording of changes in pain in VAS and QBPDS score were taken.

\section{Data Collection:-}

Base data was collected initially before the intervention, post treatment data was collected after 4 weeks of intervention and post treatment data-2 was collected after another four weeks of non-intervention.

\section{Data Analysis:-}

Statistical data analysis of the obtained values of VAS, Modified Scobers and Quebec Pain Scale Disability Questionnaire was performed using SPSS version 23.0.

A repeated measures ANOVA was performed with a Greenhouse-Geisser correction. Pair wise comparison was done using Bonferroni post hoc test. 


\section{Results:-}

Figure-1:-Pain measured by VAS

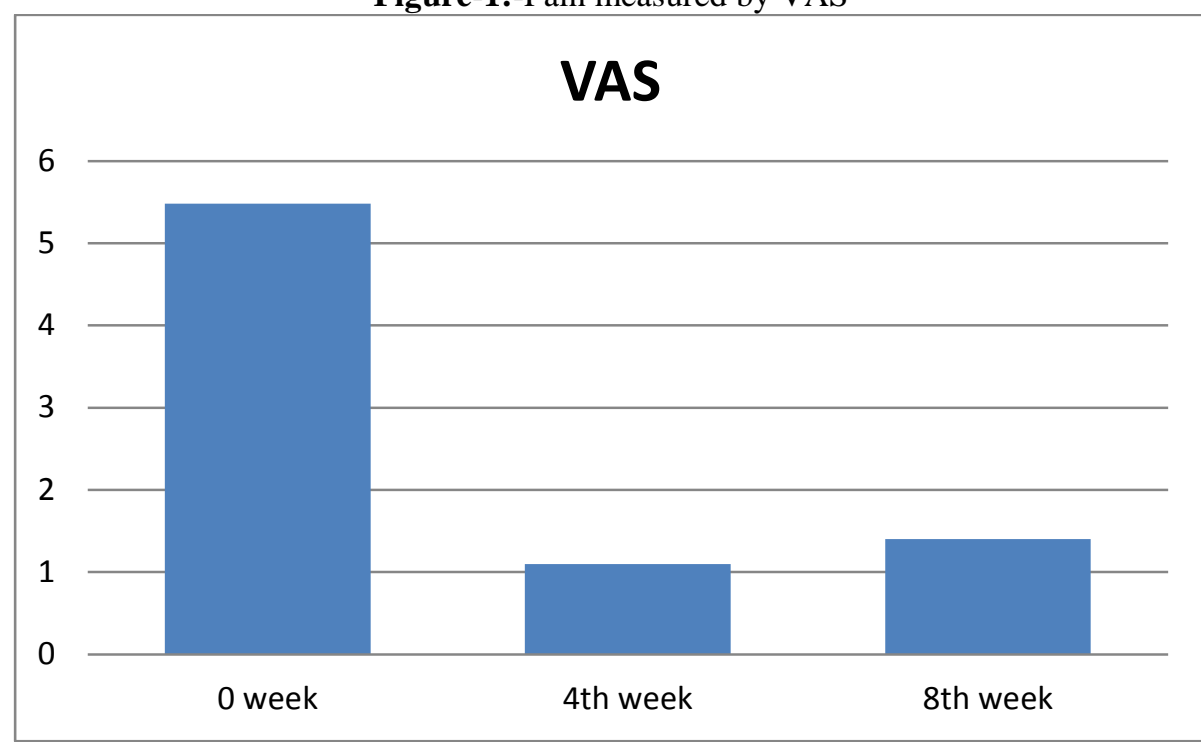

Figure-I illustrated that there was improvement in pain score measured by VAS from pre-intervention to postintervention measurements and it almost remained unchanged even after a period of another 4 weeks on nonintervention.

A repeated measures ANOVA with a Greenhouse-Geisser correction determined that mean VAS differed statistically significantly between time points $\mathrm{F}(1.293,63.349)=473.252$, $(\mathrm{P}<0.0005)$. Post hoc tests using the Bonferroni correction revealed that swimming elicited a significant reduction in VAS Scale from pre training to 4 weeks of training $(5.48+/-1.42$ vs $1.10+/-1.05$, respectively), which was statistically significant $(\mathrm{p}=.00)$. However, 4 weeks post training VAS had mildly increased to $1.40=+/-1.04$, which was statistically significantly different to pre training $(\mathrm{p}<0.0005)$ and 4 weeks training $(\mathrm{p}=0.00)$ concentrations. Therefore, we can conclude that swimming not only elicits a statistically significant reduction in VAS after 4 weeks of training, but also has some carry over effect even after 4 more consecutive weeks of non training.

Figure-2:-Modified Schober's Test (Extension)

\section{Modified Schober's Test (extension)}

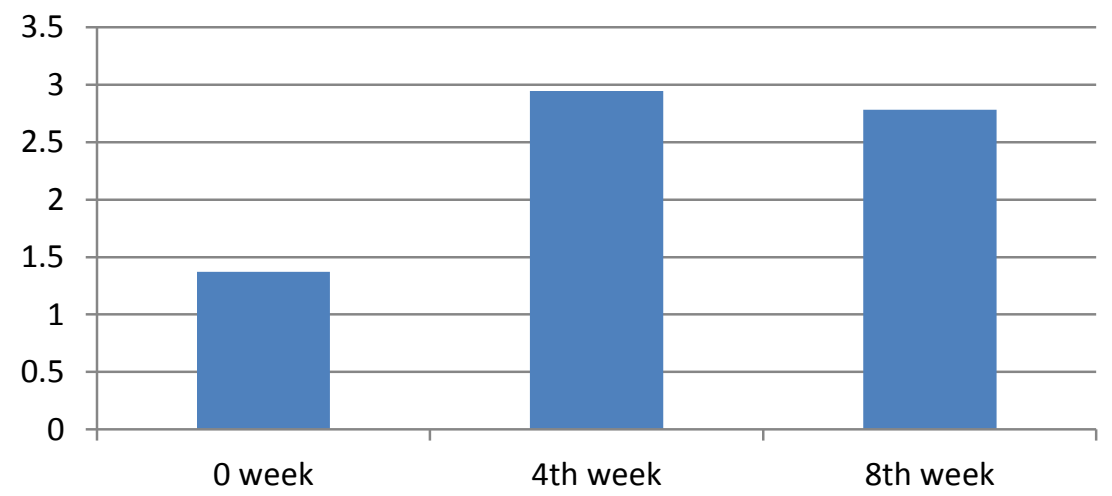


Figure-II illustrated that there was improvement in lumbar extension range of motion as measured by Modified Schober's Test from pre-intervention to post-intervention measurements and it almost remained unchanged even after a period of another 4 weeks on non-intervention.

A repeated measures ANOVA with a Greenhouse-Geisser correction determined that mean lumbar spine extension range measured by Modified Scober's Test differed statistically significantly between time points $(\mathrm{F}(1.138,55.757)$ $=174.899, \mathrm{P}<0.0005)$. Post hoc tests using the Bonferroni correction revealed that swimming elicited a significant increase in Modified Scober's Test (extension) from pre training to 4 weeks of training (1. 37 +/- 0.88 vs $2.95+/-$ 0.94, respectively), which was statistically significant. $(\mathrm{p}=.00)$. However, 4 weeks post training MST (extension) had been slightly decreased to $2.78=+/-0.98$, which was statistically significantly different to pre training $(\mathrm{p}<0.0005)$ and 4 weeks training $(\mathrm{p}=0.00)$ concentrations. Therefore, we can conclude that swimming not only elicits a statistically significant reduction in VAS concentration after 4 weeks of training, but also has some carry over effect even after 4 more consecutive weeks of non training.

Figure-3:-Modified Schober's Test (Flexion)

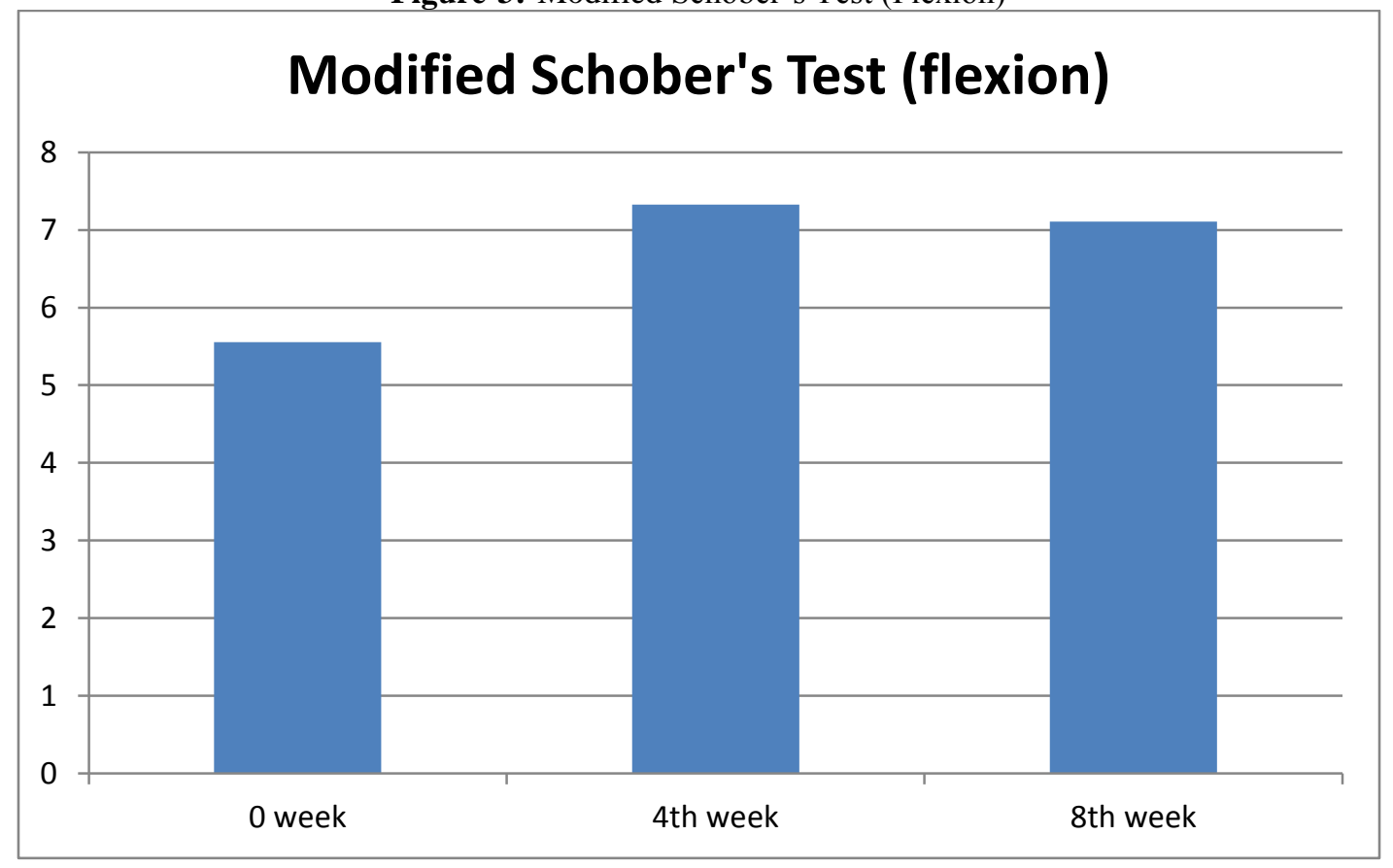

Figure-III illustrated that there was improvement in lumbar flexion range of motion as measured by Modified Schober's Test from pre-intervention to post-intervention measurements and it almost remained unchanged even after a period of another 4 weeks on non-intervention.

A repeated measures ANOVA with a Greenhouse-Geisser correction determined that mean VAS differed statistically significantly between time points $(\mathrm{F}(1.285,62.945)=189.955, \mathrm{P}<0.0005)$. Post hoc tests using the Bonferroni correction revealed that swimming elicited a significant increase in Modified Scober's Test (flexion) from pre training to 4 weeks of training $(5.55+/-0.99$ vs $7.32+/-0.66$, respectively), which was statistically significant $(\mathrm{p}=.00)$. However, 4 weeks post training MST (flexion) had been slightly decreased to $7.10=+/-0.71$, which was statistically significantly different to pre training $(\mathrm{p}<0.0005)$ and 4 weeks training $(\mathrm{p}=0.00)$ concentrations. Therefore, we can conclude that swimming not only elicits a statistically significant reduction in MST (flexion) concentration after 4 weeks of training, but also has some carry over effect even after 4 more consecutive weeks of non training. 
Figure-4:- Quebec Scale

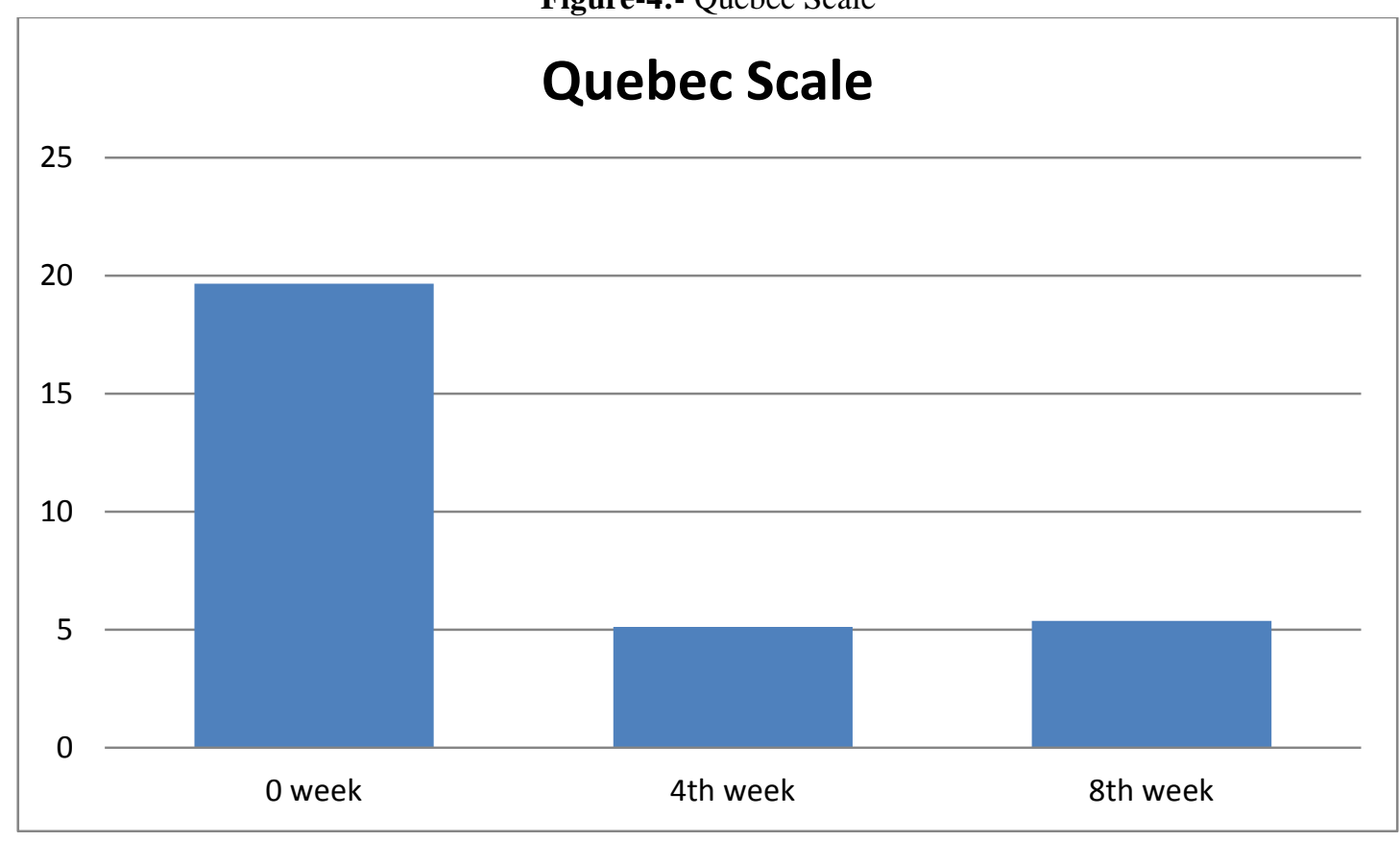

Figure- IV illustrated that there was improvement in functions measured by Quebec Back Pain Disability Questionnaire from pre-intervention to post-intervention measurements and remained unchanged even after a period of another 4 weeks on non-intervention.

A repeated measures ANOVA with a Greenhouse-Geisser correction determined that mean QBPDS differed statistically significantly between time points $\mathrm{F}(1.009,49.454)=148.397, \mathrm{P}<0.0005)$. Post hoc tests using the Bonferroni correction revealed that swimming elicited a significant reduction in Quebec Back Pain Disability Scale from pre training to 4 weeks of training $(19.67+/-9.91$ vs $5.12+/-4.83$, respectively), which was statistically significant. $(\mathrm{p}=.00)$. However, 4 weeks post training QBPDS had been mildly increased to $5.38=/-4.78$, which was statistically significantly different to pre training $(\mathrm{p}<0.0005)$ and 4 weeks training $(\mathrm{p}=0.00)$ concentrations. Therefore, we can conclude that swimming not only elicits a statistically significant reduction in QBPDS concentration after 4 weeks of training, but also has some carry over effect even after 4 more consecutive weeks of non training.

\section{Discussion:-}

The mean reduction in VAS in the subjects after the fourth and eight weeks were $4.38(79.92 \%)$ and $4.12(74.45 \%)$ respectively.

McKenzie states the third predisposing factor to low back pain is the frequency of flexion. [19] This study requires a continuous maintenance of the lumbar spine in extension position so as to keep afloat in water while swimming, which may have attributed to the increase in the range of motion of the lumbar spine.This reduces the stress on the posterior annular wall and simultaneously cause the fluid nucleus to move anteriorly - that is, away from the site of most protrusions and extrusions. [20]

\section{Stabilization Exercises:-}

According to the biomechanical model theory, weakened muscles cause mechanical irritation in the lumbar spine, thereby causing pain by stimulating pain-sensitive structures. [21] Such continued stimulation serves as an initial cause of central sensitization and chronic pain. [22] Here in this study, subjects when undergo swimming, recruit all the core muscles in order to keep the lower extremity afloat as the buoyancy acting on the centre of mass acts mostly on the upper part of the body. 
Typically, during the performance of a specific stabilization exercise, patients learn how to recruit the deep muscles of the spine and gradually reduce undesirable excessive activity of other muscles. [23] Another benefit of the core exercise program is the restoration of coordination and control of the trunk muscles to improve control of the lumbar spine and pelvis. [24] As the protective spasm reduced and muscle strength increased, the restriction of trunk movement got reduced and in turn the active spinal flexion and extension range of motions improved, in turn reducing the pain in those range of motions.

\section{Muscle strength and flexibility:-}

Muscle strength and flexibility are important for stabilizing the spine. Long-term non-use weakens these spinestabilizing muscles. The abdominal muscles and the vertebral portion of the psoas muscles are involved in producing normal vertebral alignment. [25] When exercising in water, buoyancy and the frictional resistance of water have particular mechanical effects on the body. Furthermore, fluid pressure is exerted equally on all surface areas of a stationary immersed body at a given depth. Since the impact load acting on the spine during exercises can be easily controlled by changing the extent of submersion in water, exercising in water may be the most advantageous mode of exercise for individuals with low-back pain. [26] strengthening done in such controlled environment targeted to specific muscles like the abdominal muscles, and psoas muscles, recruiting the extensors all throughout, in a high intensity, low impact exercise regimen like swimming, where extension of the spine is predominantly maintained throughout, it reverses the effect of the prolonged flexion and subsequent deconditioning leading to a pain free low back after a five days session for four weeks.

\section{Core Muscles Strengthening:-}

Swimmers need to keep their centre of mass as high as possible in the water. This requires activation of the core in all swimming strokes to prevent collapsing of the lumbar spine and pelvis. In addition forces for strong kicks are originated from core muscles. Core stabilization exercises strengthen the local stabilizers and deep muscles of the back, enhance coordination, enhance trunk stabilization and reduce the pressure on spine and subsequently reduce the low back pain. Core stabilization exercises with maximal or submaximal effort reverse the selective atrophy of type II in multifidus muscle and affect the diameter of muscular fibres. [27] The increased strength and stability facilitates a painfree movemnent of the spine.

\section{Muscle Endurance:-}

Most of the subjects recruited in this study usually had a chief complaint of back pain after prolong standing, which may be attributed to fatigue in the hip abductors and co activation in hip and truck muscles. [28] Swimming might have attributed to increasing the endurance of these muscle groups, which would have in turn resulted in reducing the pain considerably.

It should be noted that most subjects participating in the study had experienced lower back pain for more than a year. Most, having tried many forms of treatment over this time, were sceptical about how much hydrotherapy would help them. Therefore, it is interesting to note the low drop-out rate, suggesting subjects' satisfaction with the treatment method.

\section{Muscle Relaxation:-}

The heated water has a relaxing effect that can contribute in the reduction of the activation of paravertebral muscles and consequently reduce the low back pain and improve the functionality. In addition pain reduction can also be due to the action of the physical properties of water. The hydrostatic pressure (Pascal's Principle), for the action of body support and movement assistance, promotes less effort to maintain posture thus reducing muscular tone and inducing muscular relaxation. [15].

Furthermore, the treatments for low back pain in aquatic environment can be done with less risk of damage such as gravitational overload incidence of the spine, due to the action of the thrust (Archimedes's Principle), which promotes reduction in apparent weight and so can reduce muscle spasm. [15] The Ai Chi technique used at the end of every session as a cool down exercise regimen helps in relaxation in general using the principles of taichi and shiatsu and watsu, this usually helps in stabilization of the trunk, pain control, and improvement of metabolism and blood circulation. Aichi technique has been studied by numerous researchers who found it an effective tool in inducing relaxation, decreasing pain and depression in a variety of patients including chronic low back pain. [29] 


\section{Range Of Motion:-}

In the present study there was significant improvement in both the Range of Motions of lumbar spine i.e flexion and extension, after four weeks, by the end of the study. The mean improvement in lumbar flexion and extension was $1.77 \mathrm{~cm}(31.87 \%)$ and $1.58 \mathrm{~cm}(115.04 \%)$ respectively after four weeks of intervention and was $1.58 \mathrm{~cm}(27.98 \%)$ and $1.41 \mathrm{~cm}(102.92 \%)$ respectively after four more weeks of non intervention.

Limitation of lumbar extension in patients with LBP is reported by McKenzie (2003), as a common finding in patients with LBP and he advocates the use of treatment procedures that move the lumbar spine into extension to assist with the resolution of symptoms.

Reduced lumbar ROM might be the result of shortened soft tissue i.e., adaptive shortening of soft tissue and a partial loss of movement of the lumbar spine due to poor posture adapted by the chronic low back pain patients.

This study requires a continuous maintenance of the lumbar spine in extension position so as to keep afloat in water while swimming, which may have attributed to the increase in the range of motion of the lumbar spine.

The improvement in the flexion range might be attributed to elongation of the hip flexors. The spine resembles an inverted pendulum that supports the weight of the upper body; its stability requires a moving base that is provided by the joints of the lower limb, esp. the hip. [30] Clinical signs associated with low back pain are disorientation of the pelvis and the weakness or tightness of muscles around the hips.In the presence of inadequacy of the compensatory mechanisms for contracture, shortening or failure of adaptive lengthening of the psoas, and attended by weakness of the muscle, psoas insufficiency exists and the resultant shortening the thoraco-lumbar paravertebral musculature and fascia occur. The intramuscular perivascular nociceptors in the paraspinal and accessory spinal muscles and those in the ligament and fascia are thus facilitated.

This study requires subjects to swim for a considerable amount of time that is for thirty minutes, for 5 days a week, in a position that not only facilitates spinal extension but also hip extension. In an attempt to keep the lower limbs afloat extension of the hip joint is indispensible, that in turn stretches the illiopsoas.

\section{Function (Quebec Back Pain Disability Scale):-}

In the present study, towards the end of the intervention, the subjects showed reduction in Quebec Pain Scale. After completion of the study all the subjects showed significant improvement in QBPDS with the mean improvement being $14.54(73.95 \%)$ after 4 weeks of intervention and $14.28(72.63 \%)$ after another 4 weeks of non intervention. The improvement over time was significantly different from the initial stage, which maintained itself even after another four weeks of non intervention. The difference in improvement of QBPDS was better immediately after the intervention though there is a statistically significance increase in the pain scale after the period of non intervention, the scale is practically less as compared with their initial status of low back pain disability scale, which suggests a carryover effect of the intervention.

We know function is inversely proportional to pain intensity and directly proportional to the range of motion achieved. This pain discourages people from moving their affected body parts; reduction in physical activity then leads to reduced strength and reduced range of motion. [31] Normal pain free ROM is essential for normal function. This holds true for any joint in the body \& accordingly for the lumbar spine. The component of Quebec back pain disability questionnaire viz. pain intensity, personal care, lifting, walking, sitting, standing, sleeping, social life, travelling \& employment are directly related to patient's pain, and how it affects their quality of life. The reduction in QBPDS scores seen in all the subjects may be due to reduction of pain along with improvement in ROM. Higher effects on pain tends to be paired with higher use of physical function. The more reduction in QPS score, the more increase in ROM and the more the reduction of pain, the more the improvement of function.

\section{Conclusion:-}

This study found that swimming is a useful hydrotherapeutic intervention for subjects with chronic non specific low back pain as it improves range of motions, function, reduces pain and disability thus improving the quality of life of the subjects. 


\section{Limitations:-}

Sample was small, no control group was included.

\section{References:-}

1. Hoy D, March L et al. (2014) The global burden of low back pain: estimates from the Global Burden of Disease 2010 study. Ann Rheum Dis. 73:968-74

2. BenDebba M, Torgerson WS, Long DM (1997) Personality traits, pain duration and severity, functional impairment, and psychological distress in patients with persistent low back pain. Pain. 72:115-25.

3. Krismer M, van Tulder M (2007) Strategies for prevention and management of musculoskeletal conditions. Low back pain (non-specific). Best Pract Res ClinRheumatol. 21(1):77-91.

4. Bogduk N (2004) Management of chronic low back pain. MJA. 180:79-83

5. Van Tulder $\mathrm{M}$ et al. (2006) European guidelines for the management of acute nonspecific low back pain in primary care. Eur Spine J. 15:S169-S191.

6. Michel A, Kohlmann T, Raspe H (1997) The association between clinical findings on physical examination and self-reported severity in back pain. Spine. 22:296-304.

7. Von Korff M, Saunders K (1976) The course of back pain in primary care. Spine. 21(24): 2833 - 7.

8. Smith C, Grimmer-Somers K (2010) The treatment effect of exercise programmes for chronic low back pain. 16(3): $484-91$.

9. Bindra S, Sinha A, Benjamin AI (2013) Questionnaire for low back pain in the garment industry workers. Indian J Occup Environ Med. 17(2): 48 - 57.

10. Cole AJ, Becker BE (2003) Comprehensive Aquatic Therapy. $2^{\text {nd }}$ edition. Butterworth-Heinemann.

11. Harrison RA, Hillman MA, Bulstrode S (1992) Loading of the Lower Limb when Walking Partially Immersed: Implications for Clinical Practice. Physiotherapy. 78(3): $164-66$.

12. Stolberg A, Frenk H (1995) Development of water- immersion produced analgesia. Developmental Psychobiology. 28(4): $247-55$.

13. Wang TJ, Belza B, Elaine Thompson F, Whitney JD, Bennett K (2007) Effects of aquatic exercise on flexibility, strength and aerobic fitness in adults with osteoarthritis of the hip or knee. J AdvNurs. 57(2): 141 52 .

14. Dundar U, Solak O, Yigit I, Evcik D, Kavuncu V (2009) Clinical effectiveness of aquatic exercise to treat chronic low back pain: a randomized controlled trial. Spine. 34(14): 1436 - 40.

15. Konlian C (1999)Aquatic therapy: making a wave in the treatment of low back injuries. OrthopNurs. 18(1): 11 -8 .

16. Kim YS, Park J, Shim JK (2010) Effects of Aquatic Backward Locomotion Exercise and Progressive Resistance Exercise on Lumbar Extension Strength in Patients Who Have Undergone Lumbar Diskectomy. Achieves of Physical Medicine and Rehabilitation. 91(2): 208 - 14.

17. Suomi R, Lindauer S (1997) Effectiveness of Arthritis Foundation Aquatic Program on Strength and Range of Motion in Women with Arthritis. Human Kinetic Journals. 5(4): 341 - 51.

18. Gappmaier E, Lake W, Nelson AG, Fisher AG (2006) Aerobic exercise in water versus walking on land: effects on indices of fat reduction and weight loss of obese women. J Sports Med Phys Fitness. 46(4): 564 - 9.

19. McKenzie R, May S (2003) Mechanical Diagnosis and Therapy. Waikanae, New Zealand: Spinal Publications.

20. Armstrong JR (1952) Lumbar Disc Lesions. Edinburgh-London. E \& S Livingstone LTD.

21. Panjabi MM (1992) The stabilizing system of the spine. Part I. Function, dysfunction, adaptation, and enhancement. J Spinal Disord. 5(4): 383 - 9.

22. Woolf CJ (2011) Central sensitization: implications for the diagnosis and treatment of pain. Pain. 152(3 Suppl): $\mathrm{S} 2-15$.

23. Hides JA, Jull GA, Richardson CA (2001) Long-term effects of specific stabilizing exercises for first-episode low back pain. Spine. 26(11): E243 - 8 .

24. Hodges PW (2003) Core stability exercise in chronic low back pain. OrthopClin North Am. 34(2): 245 - 54.

25. Nachemson A (1965) Electromyographic Studies on the Vertebral Portion of the Psoas Muscle: With Special Reference to its Stabilizing Function of the Lumbar Spine. Journal ActaOrthopaedicaScandinavica. 37(2): 177 90.

26. Nakazawa K, Yano H, Miyashita M (1994) Ground reaction forces during walking in water. Medicine and Science in Aquatic Sports. 39: 28 - 34.

27. Bergmark A (1989) Stability of the lumbar spine. A study in mechanical engineering. ActaOrthopScand Suppl. 230: $1-54$. 
28. Marshall PW, Patel H, Callaghan JP (2011) Gluteus medius strength, endurance, and co-activation in the development of low back pain during prolonged standing. Hum Mov Sci. 30(1): 63 - 73.

29. Inoue $\mathrm{M}$ et al. (2014) The efficacy of a multidisciplinary group program for patients with refractory chronic pain. Pain Res Manag. 19(6): $302-8$.

30. Mcgregor AH, Hukins DWL (2009) Lower limb involvement in spinal function and low back pain. Journal of Back and Musculoskeletal Rehabilitation. 22(4): $219-2$.

31. Suomi R, Lindauer S (1997) Effectiveness of Arthritis Foundation Aquatic Program on strength and range of motion in women with arthritis. Journal of Aging and Physical Activity. 5: 341 - 51. 